Supporting Information

\title{
Newly Designed Copolymers for Fabricating Particles with Highly Porous Architectures
}

\author{
Chia-Chen Li, ${ }^{, 1}$ Sheng Yang, ${ }^{1}$ Yu-Ju Tsou, ${ }^{1}$ Jyh-Tsung Lee, ${ }^{2,3}$ and Chang-Ju Hsieh ${ }^{2}$ \\ ${ }^{1}$ Department of Materials \& Mineral Resources Engineering, and Institute of Materials Science and \\ Engineering, National Taipei University of Technology, Taipei 10608, Taiwan \\ ${ }^{2}$ Department of Chemistry, National Sun Yat-Sen University, Kaohsiung 80424, Taiwan \\ ${ }^{3}$ Department of Medicinal and Applied Chemistry, Kaohsiung Medical University, Kaohsiung 80708, \\ Taiwan \\ *Email: ccli@ntut.edu.tw
}




\section{Interior microstructure of the PMSV porous microsphere}

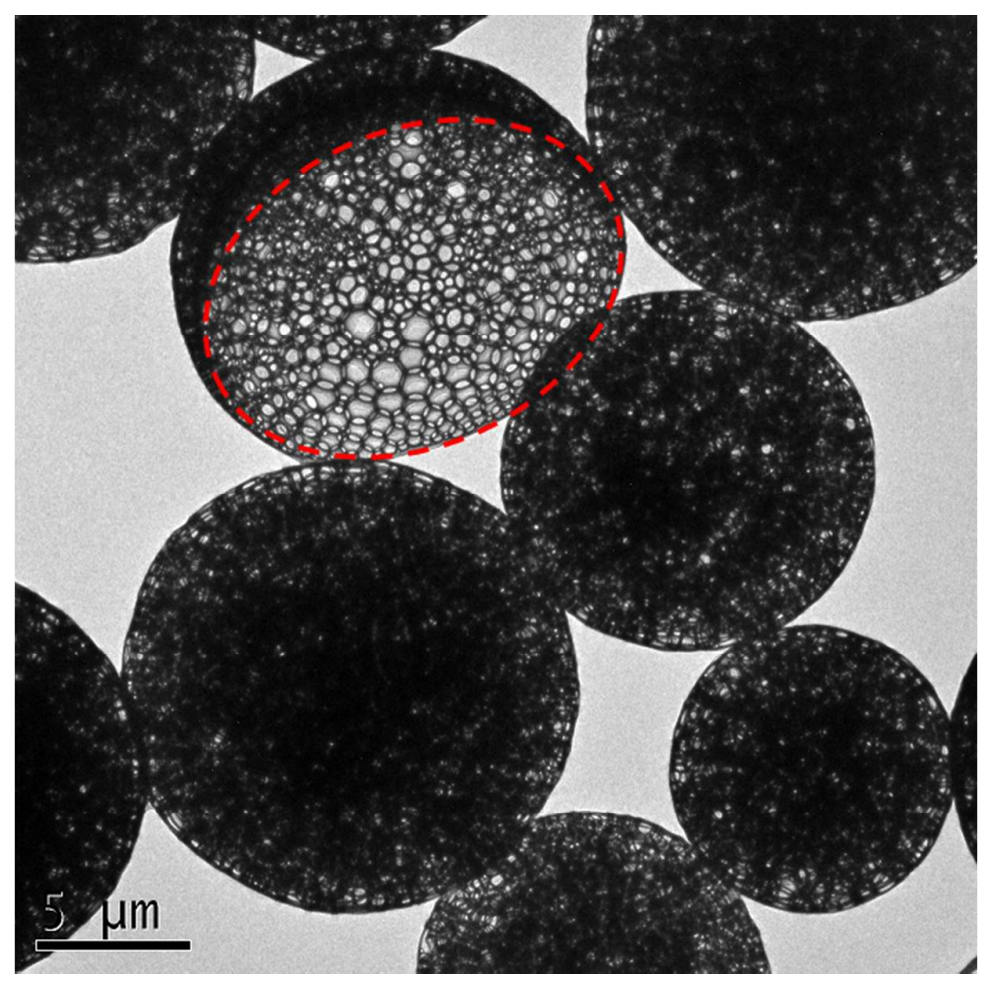

Figure S1. TEM image of porous microspheres prepared from the as-synthesized PMSV.

Compared to the microstructures of the PVBC and PSV microspheres, the PMSV microspheres exhibited a more uniform distribution of pores. Figure S1 shows that the PMSV microspheres are of the same quality and present a homogeneous morphology. The very regular distribution of pores was observed on surface and interior of the spheres, clearly shown in the cross-sectional image of the half particle in the picture (circled with a red dashed line). 


\section{Chemical characterizations for the as-synthesized PSV and PMSV}

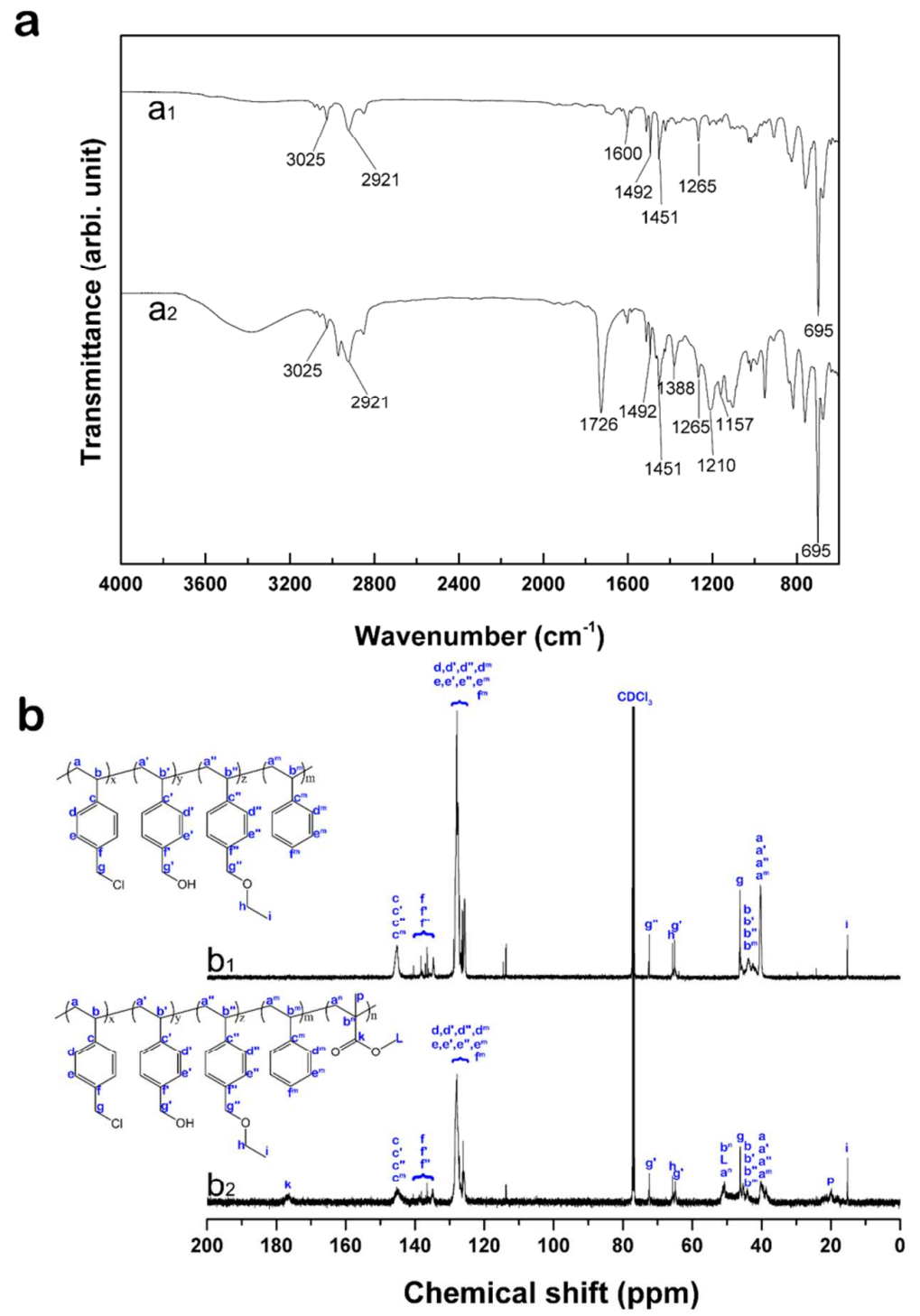

Figure S2. (a) FT-IR and (b) ${ }^{13} \mathrm{C}$ NMR spectra of the as-synthesized PSV $\left(\mathrm{a}_{1}, \mathrm{~b}_{1}\right)$ and PMSV $\left(\mathrm{a}_{2}, \mathrm{~b}_{2}\right)$.

The FT-IR spectra of the as-synthesized PSV and PMSV are shown in Figure S2(a). In both spectra, the peaks at $\sim 3000 \mathrm{~cm}^{-1}$ belong to the various $\mathrm{C}-\mathrm{H}$ stretches and the peak at $1265 \mathrm{~cm}^{-1}$ is attributed to the $-\mathrm{CH}_{2} \mathrm{Cl}$ vibration of the $\mathrm{VBC}$ unit. In addition, the broad band centered at $3380 \mathrm{~cm}^{-1}$ is attributed to the $\mathrm{O}-\mathrm{H}$ stretch. The characteristic absorption vibrations of polystyrene include the aromatic C-H stretch at $3025 \mathrm{~cm}^{-1}$, the C-H stretch at $2921 \mathrm{~cm}^{-1}$, the aromatic C-C stretches at 1600 , 
1492, and $1451 \mathrm{~cm}^{-1}$, and the aromatic C-H deformation at $695 \mathrm{~cm}^{-1}$, shown in both spectra. The characteristic IR peaks for poly(methyl methacrylate), including vibrations due to the presence of the acrylate carboxyl group at $1730 \mathrm{~cm}^{-1}, \alpha$-methyl group at $1388 \mathrm{~cm}^{-1}$, and $\mathrm{C}-\mathrm{O}-\mathrm{C}$ stretches at 1210 and $1157 \mathrm{~cm}^{-1}$, are only observed in spectrum $\mathrm{a}_{2}$. Figure $\mathrm{S} 2(\mathrm{~b})$ shows the ${ }^{13} \mathrm{C}$ NMR spectra of the as-synthesized PSV and PMSV. The chemical shifts have been assigned as shown in the figure. 


\section{Chemical characterizations for non-hydrolyzed and hydrolyzed VBC}
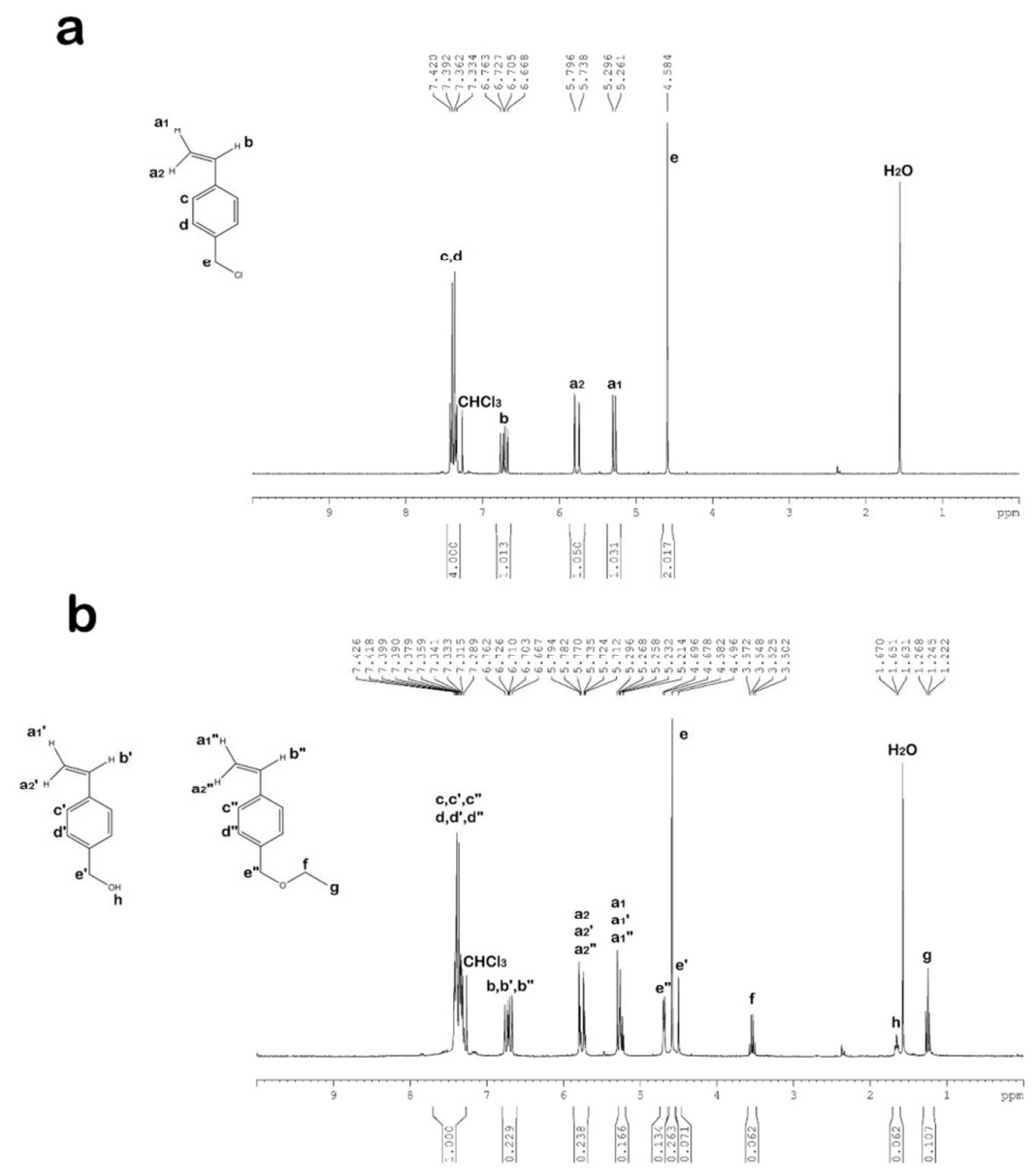

Figure S3. ${ }^{1}$ H NMR spectra of (a) as-received VBC and (b) 2 h hydrolyzed VBC.

The content and distribution of the monomer units, vinylbenzyl chloride (VBC), vinylbenzyl alcohol (VBA), and vinylbenzyl ethyl ether (VBEE), in the chemical structures of the as-synthesized copolymers were determined by the analyses of hydrolysis kinetics of VBC. The experiment for hydrolyzing $\mathrm{VBC}$ at $80{ }^{\circ} \mathrm{C}$ in the co-solvent of de-ionized water and ethanol in a volume ratio of 3:2 was carried out, and the hydrolysis products of VBC were tracked by sampling the intermediates at different time periods and analyzing the samples by ${ }^{1} \mathrm{H}$ NMR. For comparison, Figure S3(a) shows the ${ }^{1} \mathrm{H}$ NMR spectrum of the as-received VBC and the assignment for each chemical shift is shown in the figure. Figure S3(b) is the spectrum of the VBC that was hydrolyzed in the co-solvent for $2 \mathrm{~h}$. 
Three compounds, VBC, VBA, and VBEE, were observed in the spectrum. The resonances at $\sim 4.58$, 4.69, and $4.49 \mathrm{ppm}$ are assigned to the protons (e, e', e") of the methylene attached to the chlorine, hydroxyl group, and ethoxyl group, respectively. The chemical shift centered at $1.65 \mathrm{ppm}$ corresponds to the hydroxyl proton (h) and the peak centered at $3.55 \mathrm{ppm}$ corresponds to the methylene proton (f) of the ethoxyl group. The chemical shifts mentioned above are characteristic for the presence of non-hydrolyzed VBC and the hydrolyzed products VBA and VBEE. The molar percentage of these three compounds was determined via peak integration of the resonances stated above. 


\section{Chemical characterizations for PVBC}

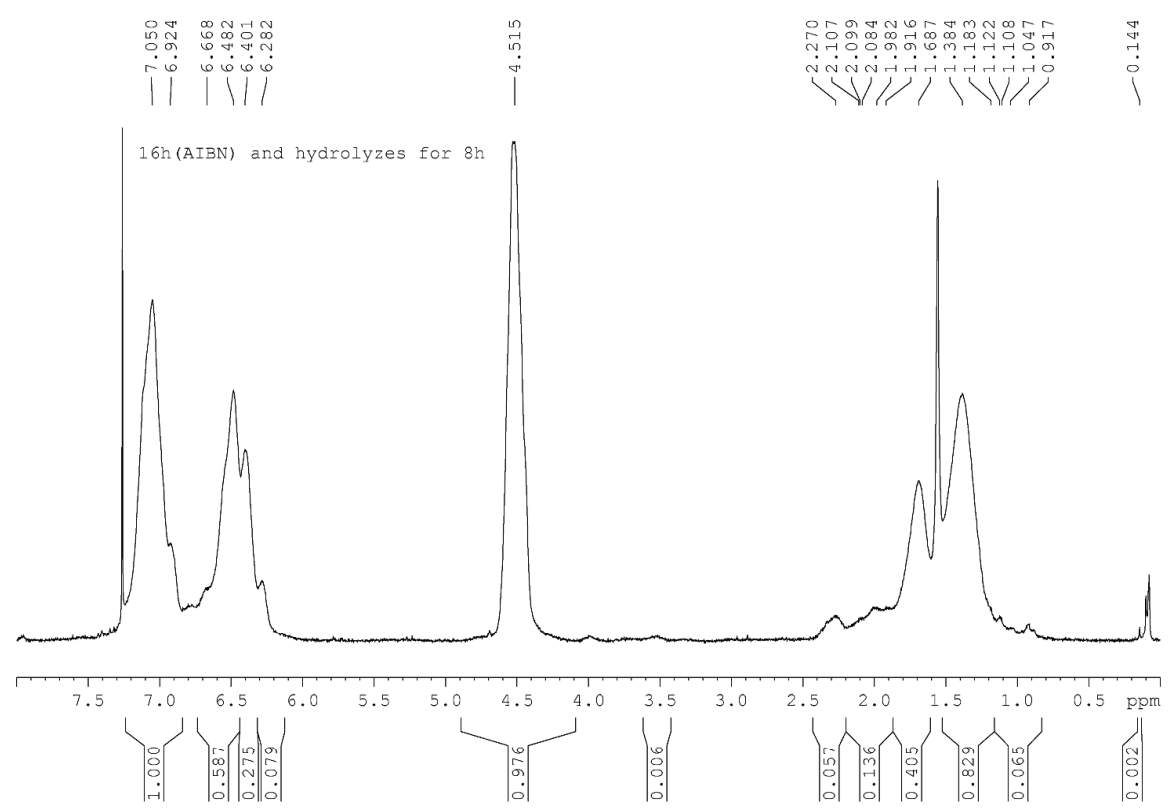

Figure S4. ${ }^{1} \mathrm{H}$ NMR spectrum of $\mathrm{PVBC}_{\alpha}$ hydrolyzed/alcoholyzed in the water/ethanol solution at 80 ${ }^{\circ} \mathrm{C}$ for $16 \mathrm{~h}$.

We used ${ }^{1} \mathrm{H}$ NMR spectroscopy to measure the hydrolysis/alcoholysis of $\mathrm{PVBC}_{\alpha}$ in the co-solvent of water/ethanol (3:2 in $\mathrm{v} / \mathrm{v})$ for $16 \mathrm{~h}$. Unlike $\mathrm{VBC}$, the hydrolysis of $\mathrm{PVBC}_{\alpha}$ was not easy to be identified because the benzylic hydrogens at 4.2-4.8 ppm were overlapped in the ${ }^{1} \mathrm{H}$ NMR spectrum of polymers. However, the alcoholysis of PVBC can be characterized directly by the ratio of peaks at $3.5\left(-\mathrm{OCH}_{2} \mathrm{CH}_{3}\right)$ and $4.5\left(-\mathrm{Ph}-\underline{\mathrm{C}}_{2}-\mathrm{O}-\right)$ ppm. The ${ }^{1} \mathrm{H}$ NMR result (Figure $\left.\mathrm{S} 4\right)$ shows that the alcoholysis of $\mathrm{PVBC}_{\alpha}$ is only $0.6 \%$, which should be due to its poor solubility in the water/ethanol solution. To have a shorter PVBC with lower molecular weight, which should have better solubility in water/ethanol, we polymerized VBC in the same condition (water/ethanol solution at $80{ }^{\circ} \mathrm{C}$ ) for only $8 \mathrm{~h}$, not $16 \mathrm{~h}$. The synthesized PVBC, denoted as $\mathrm{PVBC}_{8 \mathrm{~h}}$, which ${ }^{1} \mathrm{H}$ NMR spectrum was shown in Figure S5 was purified and further heated in the water/ethanol solution at $80{ }^{\circ} \mathrm{C}$ for another $8 \mathrm{~h}$ to 
measure the alcoholysis. The ${ }^{1} \mathrm{H}$ NMR result (Figure S6) indicates that after heating in the water/ethanol solution at $80{ }^{\circ} \mathrm{C}$ for $8 \mathrm{~h}$ the percentage of alcoholysis of $\mathrm{PVBC}_{8 \mathrm{~h}}$ is $7.4 \%$, which is still much lower than the alcoholysis of VBC (38.9\%, Figure 3(a)). That is, the alcoholysis reaction of the polymer chain is significantly slower than that of the monomer, evidencing that our proposed copolymers are highly proable to be in a gradient structure.

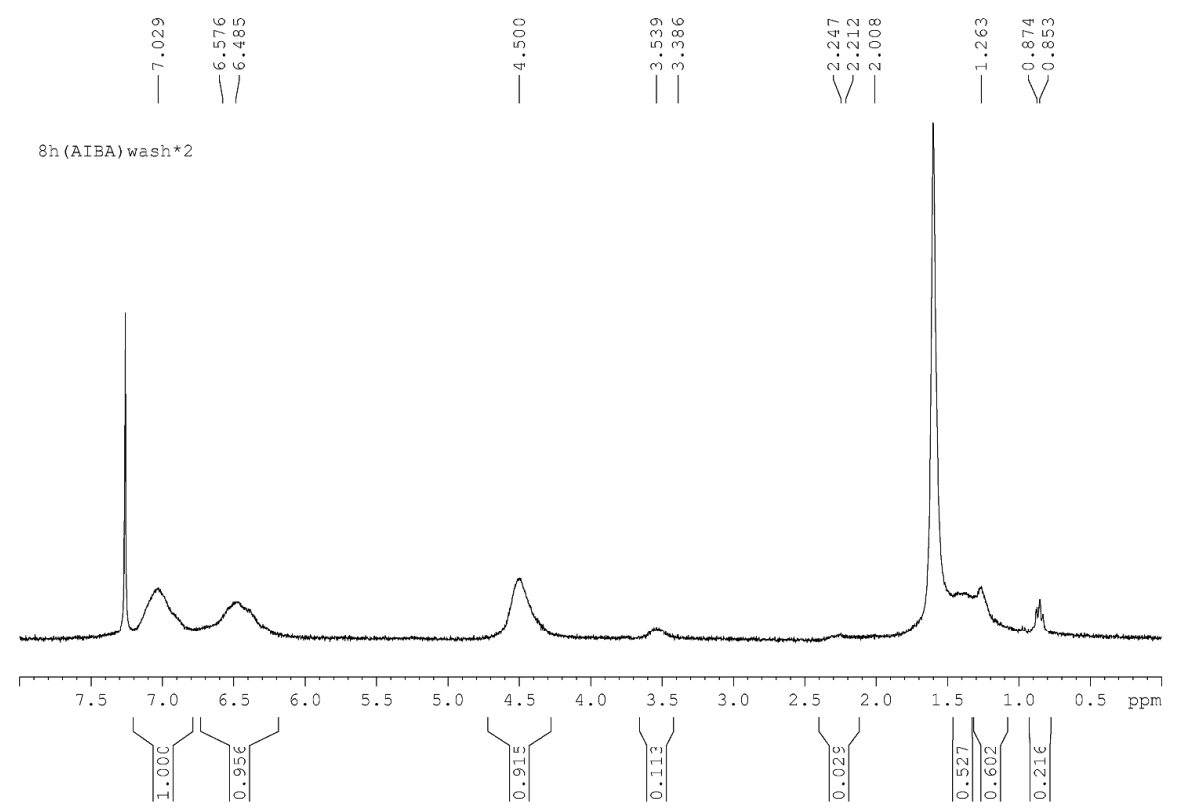

Figure S5. ${ }^{1} \mathrm{H}$ NMR spectrum of $\mathrm{PVBC}_{8 \mathrm{~h}}$. 


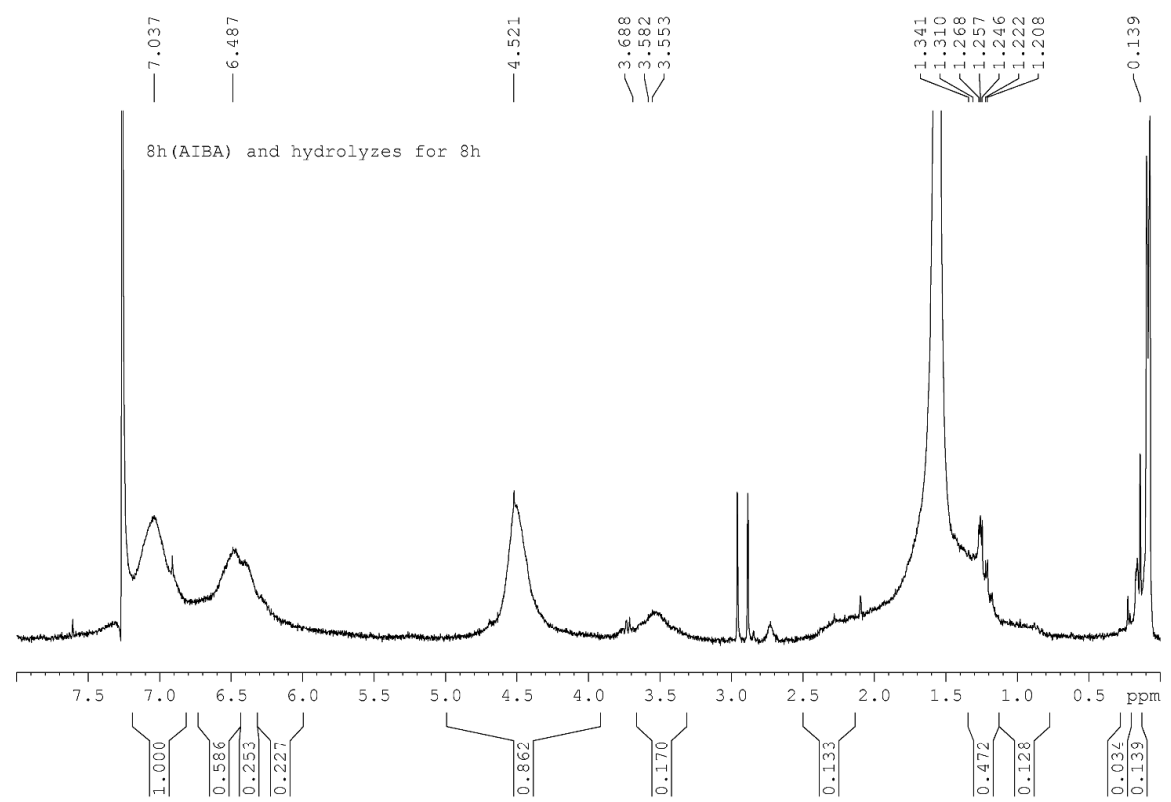

Figure S6. ${ }^{1} \mathrm{H}$ NMR spectrum of the purified and further heated $\mathrm{PVBC}_{8 \mathrm{~h}}$ in the water/ethanol solution at $80{ }^{\circ} \mathrm{C}$ for $8 \mathrm{~h}$.

Table S1. The percentage of alcoholysis of $\mathrm{VBC}, \mathrm{PVBC}_{\alpha}$, and $\mathrm{PVBC}_{8 \mathrm{~h}}$ after heating in the water/ethanol solution at $80{ }^{\circ} \mathrm{C}$.

\begin{tabular}{lc}
\hline & Percentage of alcoholysis (\%) \\
\hline VBC & 38.9 \\
PVBC $_{\boldsymbol{\alpha}}$ & 0.6 \\
$\mathbf{P V B C}_{\mathbf{8 h}}$ & 7.4 \\
\hline
\end{tabular}




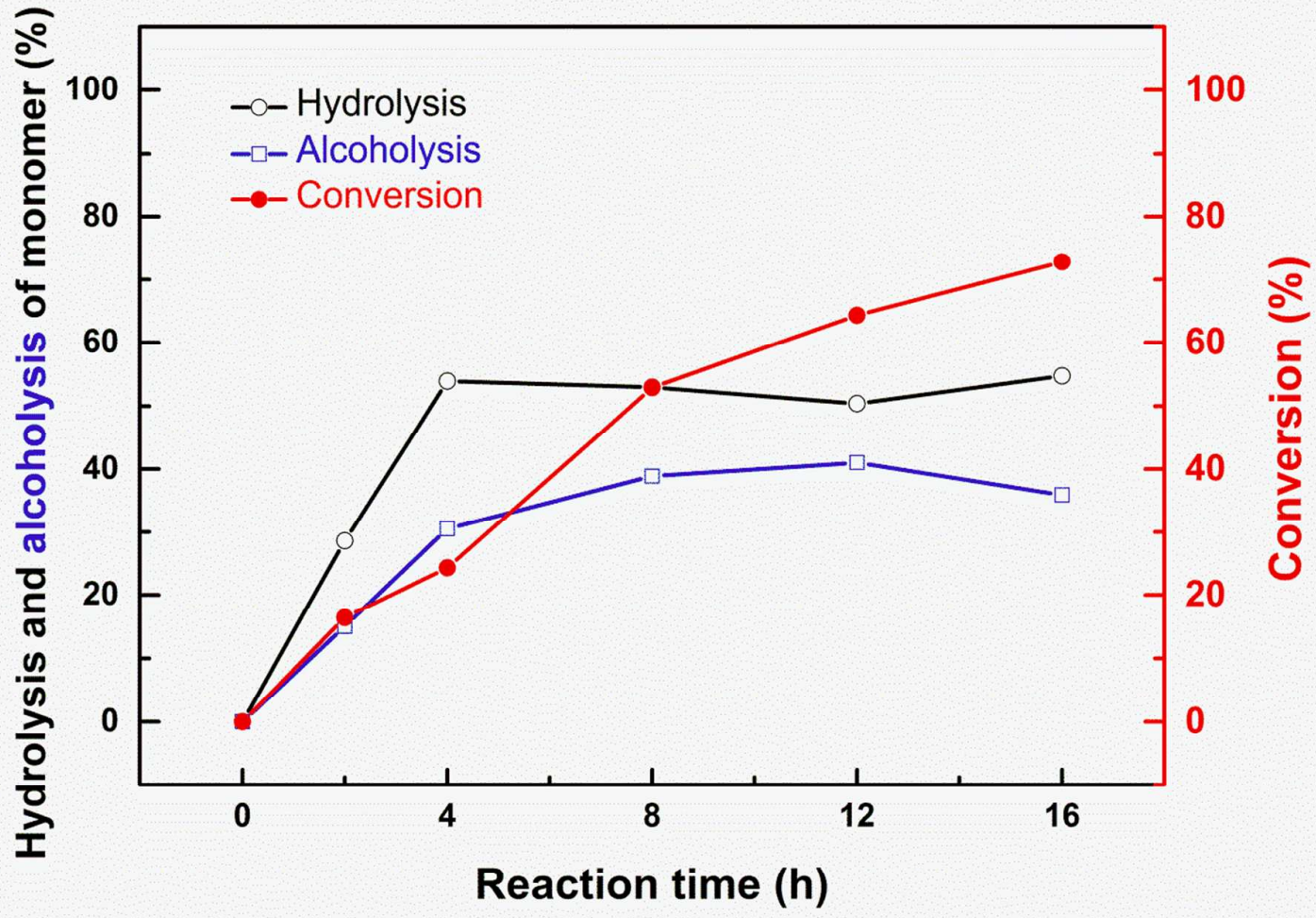

Figure S7. Hydrolysis and alcoholysis of the VBC monomer and conversion of polymerization as a function of reaction time in the water/ethanol solution at $80{ }^{\circ} \mathrm{C}$.

In Figure S7, we found that the rate of polymerization is slower than the rate of hydrolysis/alcohol of monomer. At the beginning, the major monomer is VBC. During the polymerization of VBC, the hydrolysis/alcohol of the VBC monomer also proceeds, and thus the content of hydrolyzed/alcoholized VBC monomers will increase as polymerization proceeds. Therefore, the polymer chains of as-synthesized polymers should contain higher content of hydrophobic VBC unit in the beginning of the polymerization, and higher content of hydrophilic VBA units as polymerization proceeds. 


\section{Microspheres prepared from a random copolymer of $\mathrm{VBC}, \mathrm{VBA}$, and VBEE}

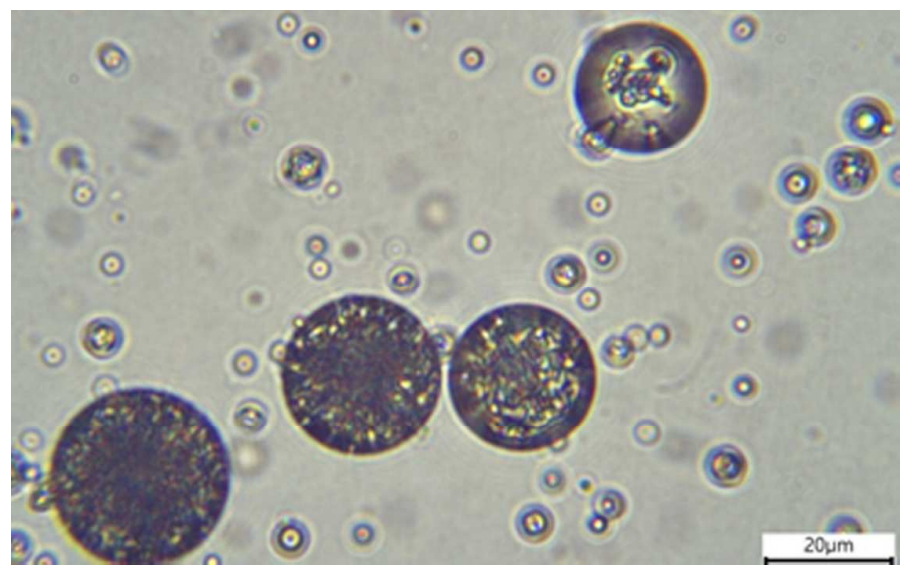

Figure S8. OM image of the microspheres prepared from a random copolymer of VBC, VBA, and VBEE.

A random copolymer containing monomer units of VBC, VBA, and VBEE was synthesized. Before polymerization, the VBC monomer was pre-hydrolyzed in a co-solvent of water/ethanol (3:2 in $\mathrm{v} / \mathrm{v}$ ) at $80{ }^{\circ} \mathrm{C}$ for $16 \mathrm{~h}$. Then, the hydrolyzed products were separated from the co-solvent and placed in toluene. Next, polymerization proceeded at $80{ }^{\circ} \mathrm{C}$ for $16 \mathrm{~h}$ with the addition of the organic-based initiator 2,2'-azobis(2-methylpropionitrile) (AIBN; 98\%, Sigma-Aldrich, Saint Louis, USA). As the non-hydrolyzed VBC and the hydrolyzed monomers, VBA and VBEE, are expected to have similar reaction rates, the synthesized copolymer likely possesses a homogeneous distribution of VBC, VBA, and VBEE in the polymerized chain. For fabrication of microspheres, the copolymer was dissolved in toluene and then emulsified in an aqueous solution containing $0.3 \mathrm{wt} . \%$ of SDS under a homogenizing speed of $3400 \mathrm{rpm}$ for 2 min. Figure S8 shows the optical microscopic (OM) image of the resulting microspheres, demonstrating that the interior structure should be porous. This result is different from those obtained when the copolymers were synthesized by in-situ hydrolysis. This result indicates the distribution of monomer units may vary the physicochemical properties of the synthesized copolymers and thus, influence the architectural quality of the formed microspheres. 